\title{
Características individuais associadas à autopercepção da extensão territorial da vizinhança
}

\author{
Individual characteristics associated with \\ perception of the local neighborhood's \\ territory
}

\section{Las características individuales asociadas con la percepción de la extensión territorial del vecindario}

\author{
1 Universidade Federal de \\ Minas Gerais, Belo Horizonte, \\ Brasil. \\ 2 Secretaria Estadual de \\ Saúde de Minas Gerais, Belo \\ Horizonte, Brasil. \\ 3 Faculdade de Saúe \\ e Ecologia Humana \\ Vespasiano, Brasil. \\ ${ }^{4}$ Centro de Pesquisas René \\ Rachou, Fundação Oswaldo \\ Cruz, Belo Horizonte, Brasil. \\ Correspondência \\ F. A. Célio \\ Universidade Federal de \\ Minas Gerais. \\ Rua Ipatinga, 270, Sete \\ Lagoas, $M G$ \\ 35701-059, Brasil. \\ maitiz@gmail.com
}

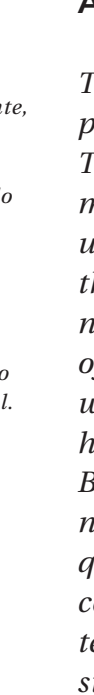




\section{Introdução}

Nas últimas décadas, verifica-se o crescente interesse em determinar e quantificar as características físicas e sociais da vizinhança que têm impacto sobre a saúde das populações ali aninhadas 1. Desfechos diversos têm sido associados a essas características, incluindo as doenças cardiovasculares 2 , doenças sexualmente transmissíveis 3 , saúde mental 4,5 e mortalidade 6 , dentre outras 7,8,9.

Para a delimitação de vizinhança, duas são as estratégias mais frequentemente encontradas na literatura. A primeira utiliza unidades geográficas administrativas, as quais podem ser mais facilmente identificadas e delimitadas. Tais unidades, geralmente definidas por órgãos administrativos (por exemplo, o setor censitário utilizado pelo Instituto Brasileiro de Geografia e Estatística - IBGE), reduzem os custos de pesquisa, facilitam a periodicidade e a obtenção de dados secundários. Uma dificuldade dessa estratégia é a possibilidade de desconsiderar a heterogeneidade física e social no interior dessas áreas, podendo resultar em viés de informação 10 .

Outra estratégia utiliza a percepção dos moradores para delimitação da vizinhança, podendo essa coincidir ou não com unidades administrativas 11,12,13,14. Apesar da dificuldade em agregar dados secundários relativos à vizinhança autopercebida 7, essa abordagem é promissora, uma vez que leva, em consideração, a extensão territorial em que o sujeito desempenha as suas atividades cotidianas e mantém os seus laços sociais. Em geral, os estudos utilizam duas estratégias: o participante delimita a sua vizinhança em um mapa 15,16 ou responde a perguntas abertas ou fechadas referentes à extensão da sua vizinhança 17,18 .

Diferentes indivíduos aninhados em uma vizinhança demarcam as suas próprias fronteiras e, para isso, consideram sua identificação social com o local, o espaço onde realizam suas atividades diárias e os locais que costumam frequen$\operatorname{tar} 17$. A percepção individual interfere diretamente na dimensão da vizinhança e como ela é compreendida; a percepção não é conhecimento, depende de interpretação ${ }^{19}$. Portanto, a percepção do tamanho de uma vizinhança pode ser influenciada por características individuais, como experiências e preferências, e contextuais 15 .

Verifica-se também a pouca padronização e validação de métodos para acessar a vizinhança autopercebida, o que dificulta sua utilização em estudos epidemiológicos.

Ainda são raros, na literatura consultada, estudos que mensuraram a extensão territorial da vizinhança autopercebida 15,16,17,18. Neste estu- do, objetivamos determinar e quantificar a associação entre a extensão da vizinhança autopercebida e as características individuais dos participantes de inquérito de saúde em Belo Horizonte.

\section{Métodos}

\section{Origem dos dados, amostragem e questionário}

Os dados deste estudo são oriundos do estudo Saúde em Beagá (SBH), nome fantasia de inquérito de saúde domiciliar realizado entre 2008 e 2009 pelo Observatório de Saúde Urbana de Belo Horizonte (OSUBH), da Universidade Federal de Minas Gerais (UFMG), em dois dos nove distritos sanitários (DS) de Belo Horizonte - Barreiro e Oeste 20,21,22 (Figura 1). Esses dois DS somam 531 mil habitantes, correspondendo a $22,4 \%$ da população da cidade e subdivididos em 568 setores censitários 20,21,22. Um dos principais de terminantes para a seleção desses DS foi o fato de apresentarem importante heterogeneidade interna em relação a diversos indicadores demográficos, socioeconômicos e de saúde.

A amostra foi selecionada por processo de estratificação em conglomerados em três estágios. Para garantir a presença proporcional de residentes em todos os níveis socioeconômicos, a área de estudo foi subdividida em estratos, de acordo com o Índice de Vulnerabilidade à Saúde (IVS - mínimo 0,25; máximo 6,35), segundo as categorias de vulnerabilidade tradicionalmente utilizadas pela Secretaria Municipal de Saúde (SMS): baixa $(<2,33)$, média $(2,33-3,32)$, elevada $(3,33-4,31)$ e muito elevada $(>4,31) 23$. O IVS é um indicador composto, criado pela Secretaria $\mathrm{Mu}$ nicipal de Saúde de Belo Horizonte (SMS-BH), que abrange aspectos relacionados a saneamento (abastecimento de água, esgotamento e destino do lixo), habitação (domicílios improvisados e número de moradores por residência), educação (analfabetismo e escolaridade do chefe da família), renda (familiar e do chefe da família), social e saúde (chefe de família adolescente, coeficiente de mortalidade em menores de cinco anos, coeficiente de óbitos cardiovasculares em pessoas de 30-59 anos e óbitos proporcionais em pessoas com menos de 70 anos). A combinação dessas informações resulta em escore para cada setor censitário. Valores elevados indicam setores censitários de alta vulnerabilidade/risco. A construção do IVS teve como um de seus objetivos avaliar diferenciais socioeconômicos intraurbanos e delimitar áreas prioritárias para intervenção e alocação de recursos. Após a subdivisão dos setores censitários aninhados nos dois DS 

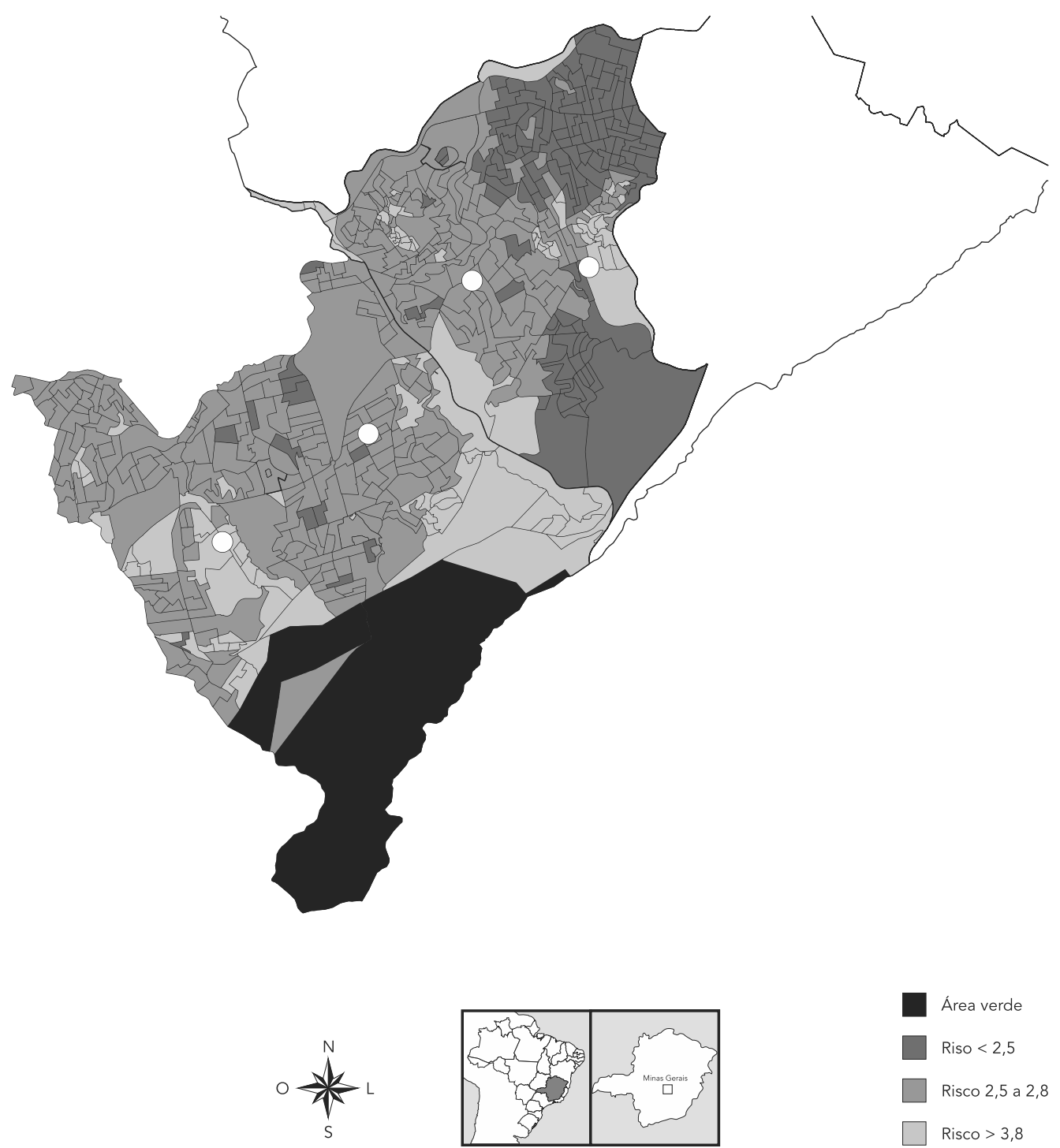

de acordo com o valor do IVS, procedeu-se da seguinte maneira: no primeiro e segundo estágios, foram selecionados 150 setores censitários, que continham 6.439 endereços elegíveis, selecionados por amostra aleatória simples. Após exclusão de lotes vagos, endereços institucionais e comerciais e aqueles nos quais moradores não foram localizados, 5.436 domicílios permaneceram como elegíveis. A proporção de recusas foi de $26 \%$, resultando em 4.048 domicílios. No terceiro estágio, um residente adulto, com 18 anos e mais, foi selecionado em cada um dos domicílios. Nas residências selecionadas, era procedido o sorteio do morador elegível. Após serem esclarecidos sobre os objetivos da pesquisa, os potenciais participantes eram convidados a assinar o termo de consentimento livre e esclarecido e a responder ao questionário. 
Variáveis

\section{- Variável resposta}

Para obtenção da variável resposta, inicialmente, foi lida, para o participante, a seguinte definição de vizinhança: "Vizinhança é o local onde o(a) sr.(a) vive e realiza tarefas de rotina tais como ir à padaria, sacolão, comércio local, visitar seus vizinhos, caminhar. Pode-se entender como vizinhança também o local onde o(a) sr. (a) reconhece a maioria das pessoas". Em seguida, era perguntado ao participante: "Pensando na sua vizinhança, o(a) senhor(a) diria que ela vai até: (1) as residências mais próximas da sua; (2) o final deste quarteirão; (3) os 5 quarteirões mais próximos; (4) os 10 quarteirões mais próximos de sua residência; (5) mais de 10 quarteirões da sua residência; (6) seu bairro/comunidade/conjunto habitacional; (7) seu bairro e bairros mais próximos".

Posteriormente, a variável resposta foi recodificada, utilizando-se, como referência, variável contínua obtida com a pergunta: "Quanto tempo em minutos o(a) senhor(a) gastaria para ir caminhando da porta da sua casa até o final do que o(a) senhor(a) considera sua vizinhança?". Valendo-se da média em minutos dessa variável para cada um dos 7 estratos iniciais da variável resposta e considerando a não sobreposição dos intervalos de 95\% de confiança (IC95\%) entre os estratos, a variável resposta foi recodificada, obtendo-se, assim, quatro estratos: (1) consideram as residências mais próximas da sua e até o final do quarteirão; (2) consideram os 5 quarteirões mais próximos; (3) consideram os 10 quarteirões mais próximos da sua residência; e (4) seu bairro/comunidade/conjunto habitacional e bairros mais próximos.

\section{- Variáveis explicativas}

As variáveis explicativas foram escolhidas segundo o modelo teórico da Figura 2, que representa a percepção da extensão territorial da vizinhança, e parcialmente contempladas nas escalas para o nível hierárquico agregado de vizinhança elaboradas por Friche et al. 22, que utilizaram a mesma base de dados deste estudo. As escalas de vizinhança, acrescida da escala participação social, foram adaptadas para serem utilizadas no nível individual. Essas escalas incorporam variáveis do contexto físico e social do local de moradia, obtidas a partir da percepção do indivíduo, como serviços, infraestrutura urbana, estética, segurança na vizinhança, dentre outros (Figura 3). As seguintes escalas recebem valores mais altos para percepção positiva dos itens constituintes delas: qualidade de serviços, aspecto estético, mobi- lidade, segurança, coesão social e participação social (indivíduos mais participativos recebem valores mais altos). As escalas desordem física, desordem social e problemas na vizinhança recebem valores altos em seu escore, com o aumento da percepção de vizinhanças mais vulneráveis.

Outras variáveis, previamente relatadas ou consideradas pelos autores como potencialmente associadas à percepção da extensão territorial da vizinhança, também foram incluídas nas análises, a saber: (a) idade em anos; (b) sexo (feminino, masculino); (c) estado civil (solteiro, separado, viúvo, casado, amigado); (d) situação ocupacional atual (não trabalha, trabalha); (e) indicador econômico nacional (IEN) 24, que foi utilizado como marcador de posição socioeconômica atual (indivíduos em posição socioeconômica menos vulnerável recebem valores mais altos para esse índice); (f) presença de crianças menores de 10 anos de idade no domicílio (não sim); (g) tempo de moradia no mesmo domicílio em anos; (h) número de parentes morando na vizinhança; (i) reconhece praticamente todos que passam na porta da sua casa (Tabela 1 ).

\section{Análise estatística}

A primeira etapa consistiu na adaptação das escalas de vizinhança para o nível hierárquico individual e na elaboração da escala de percepção do entorno físico e social da vizinhança, denominada participação social. Por meio de análise de componentes principais, foram construídos os escores das escalas. A seleção dos itens que compõem cada escala é descrita por Friche et al. 22. Os componentes foram selecionados tendo, como referência, o acúmulo de $70 \%$ da explicação da variância total. O valor do alfa de Cronbach 21 foi utilizado para avaliar a consistência interna de cada escala.

Inicialmente, para determinar a associação entre a variável resposta e as variáveis explicativas, foi realizada análise univariada utilizando o teste de qui-quadrado e regressão logística ordinal, modelo de chances proporcionais 25. Esse modelo de regressão foi escolhido dada a natureza ordinal da variável resposta, assumindo ainda proporcionalidade da odds entre os diferentes estratos dela, premissa verificada com a realização do teste de Wald 25 . As variáveis que apresentaram um valor de $\mathrm{p} \leq 0,20$ na análise univariada foram incluídas na análise multivariada. As variáveis idade, sexo, IEN e tempo em anos de residência no domicílio foram mantidas no modelo final, independentemente da significância estatística. A primeira componente principal de cada escala de vizinhança foi incluída na análise multivariada, independentemente do valor de $\mathrm{p}$ 


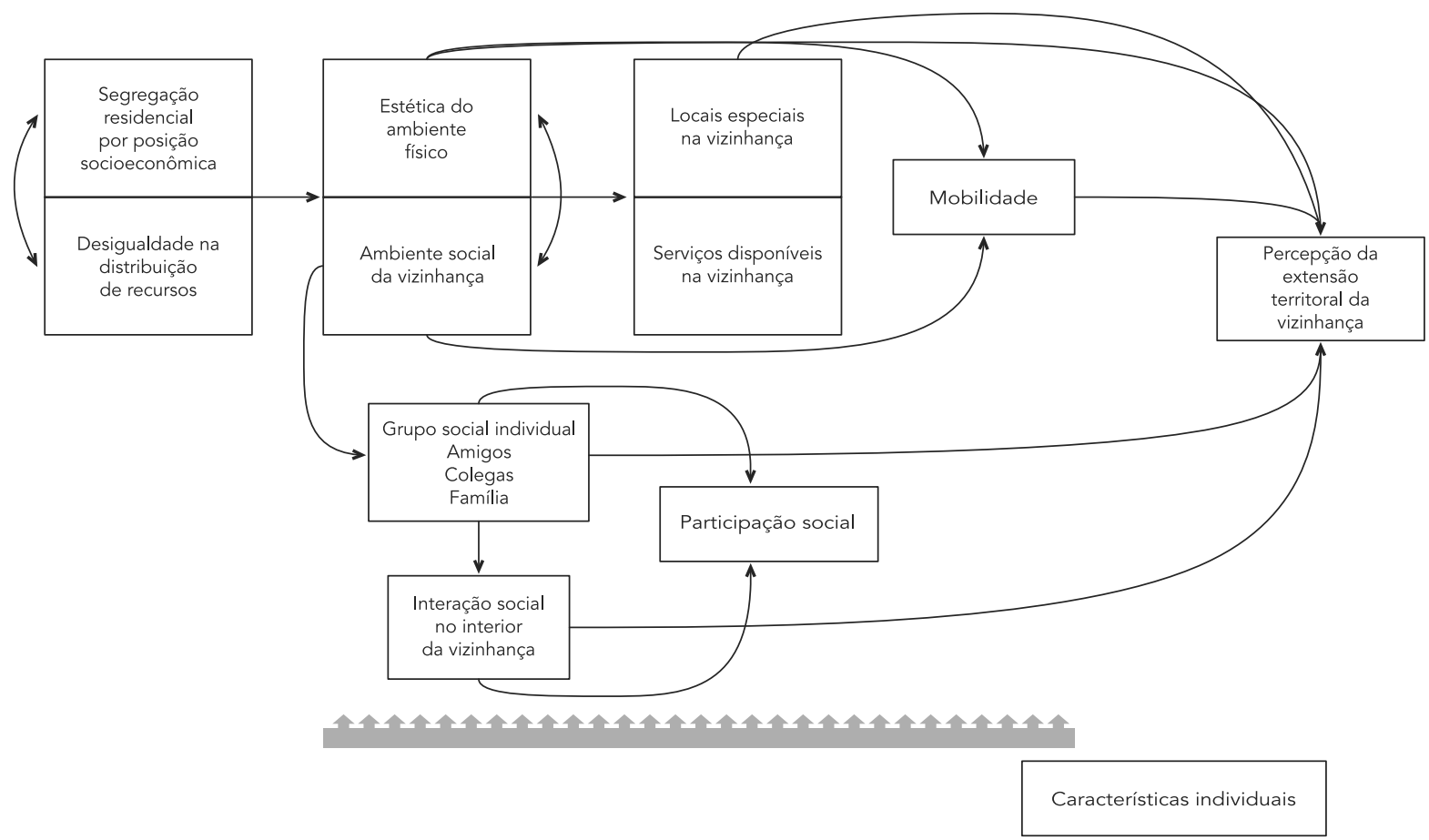

Adaptado de Diez-Roux \& Mair 32

na análise univariada. As demais variáveis com valor de $\mathrm{p} \leq 0,05$ permaneceram no modelo final. A magnitude da associação foi estimada a partir da odds ratio (OR) e seu respectivo IC95\%. O teste de regressão paralela, teste de Wald, foi realizado para verificação do modelo final, e o teste de Deviance foi utilizado para verificação do ajuste do modelo 25 . Todas as análises foram realizadas no software Stata 11 (Stata Corp., College Station, Estados Unidos). O comando $s v$ y permitiu considerar o desenho amostral nos ajustes do modelo logístico ordinal. O svy permite corrigir as estimativas dos coeficientes e respectivos erros-padrão dos modelos logísticos ao considerar as complexidades do desenho amostral (estratos e conglomerados) e ainda as diferentes probabilidades de seleção dos indivíduos resultantes da amostragem em múltiplos estágios e sobreamostragem de determinadas áreas 20.

Este estudo foi aprovado pelo Comitê de Ética em Pesquisa da UFMG (COEP - Parecer no ETIC 017/07 e Parecer no ETIC 253/006).

\section{Resultados}

A amostra final contou com 4.048 respondentes, sendo $53,1 \%$ homens e $46,9 \%$ mulheres, com idade variando entre 18 e 95 anos (média = 44,4; $\mathrm{DP}=16,9$ ). Com relação à percepção da extensão territorial da vizinhança, $57,8 \%$ dos participantes consideraram sua vizinhança como as residências mais próximas da sua e até o final do quarteirão, 23,3\% consideraram os 5 quarteirões mais próximos, 7,4\% os 10 quarteirões mais próximos de sua residência e $11,5 \%$ consideraram sua vizinhança como mais de 10 quarteirões da sua residência (Tabela 1).

Após a recodificação da variável resposta, foram obtidos os seguintes tempos médios, em minutos, para se caminhar até o final da vizinhança de acordo com os estratos da variável resposta: (1) as residências mais próximas da sua e até o final do quarteirão (média = 6,15; IC95\%: 5,716,58); (2) os 5 quarteirões mais próximos (média = 13,54; IC95\%: 12,84-14,23); (3) os 10 quarteirões mais próximos de sua residência (média $=19,76$; 
Figura 3

Descrição dos itens constituintes das escalas de percepção do entorno físico e social da vizinhança.

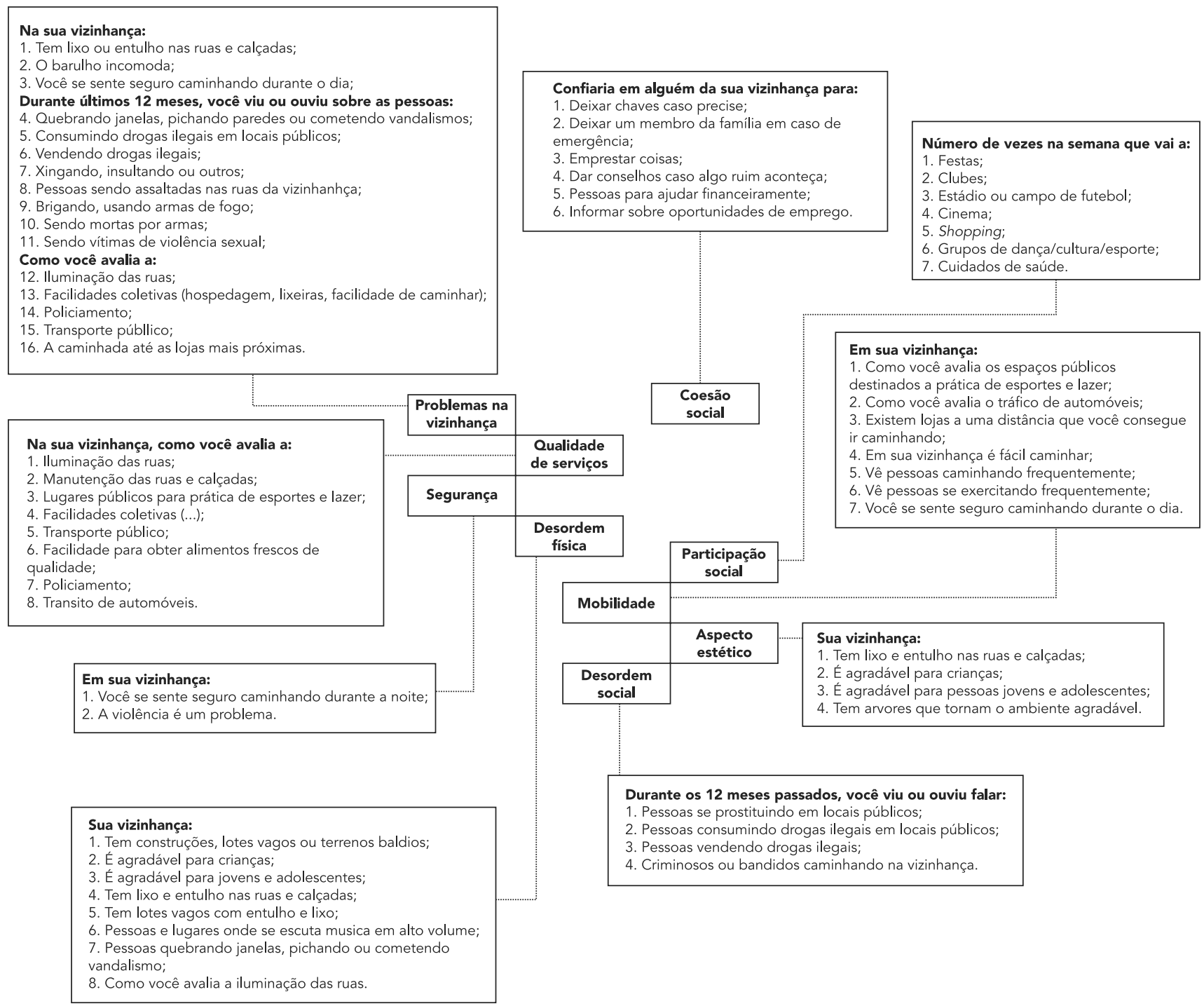

Adaptado de Friche et al. 22.

IC95\%: 18,23-21,29) e (4) mais de 10 quarteirões da sua residência; seu bairro/comunidade/conjunto habitacional e bairros mais próximos (média $=29,17$; IC95\%: 26,69-31,65).

O número de itens que compõem as escalas variou entre 4 e 16, e o resultado do alfa de Cronbach revelou consistência interna aceitável para todas as escalas utilizadas, com valor mínimo de 0,51 e valor máximo de 0,83 . Para o acúmulo de $70 \%$ da explicação da variância total, foram selecionados, no máximo, quatro componentes principais, sendo que a escala segurança necessitou de apenas um. Assumiu-se que as perdas verificadas em cada escala foram devido ao acaso (Tabela 2).

No modelo final (Tabela 3), o aumento da percepção da extensão territorial da vizinhança foi estatisticamente associado com: posição socioeconômica medida pelo IEN (OR = 1,09; IC95\%: 1,08-1,34, correspondendo ao incremento de 200 pontos do IEN); estar trabalhando (OR = 1,32; IC95\%: 1,07-1,61); avaliação positiva dos 
Distribuição de frequência e dispersão de variáveis e escalas selecionadas, estratificadas de acordo com a extensão territorial da vizinhança autopercebida para 4.048 participantes. Estudo Saúde em Beagá, Belo Horizonte, Minas Gerais, Brasil, 2008-2009.

\begin{tabular}{|c|c|c|c|c|}
\hline \multirow[t]{2}{*}{ Variável } & \multicolumn{4}{|c|}{ Extensão territorial da vizinhança (\%) * } \\
\hline & $\begin{array}{c}1 \\
(57,8 \%)\end{array}$ & $\begin{array}{c}2 \\
(23,3 \%)\end{array}$ & $\begin{array}{c}3 \\
(7,4 \%)\end{array}$ & $\begin{array}{c}4 \\
(11,5 \%)\end{array}$ \\
\hline Sexo (masculino) & 43,6 & 49,0 & 54,1 & 54,1 \\
\hline Estado civil (casado, amigado) & 54,0 & 50,2 & 44,8 & 50,0 \\
\hline Trabalha (sim) & 62,0 & 65,6 & 69,0 & 73,3 \\
\hline Presença de criança (sim) & 33,1 & 31,5 & 30,6 & 33,0 \\
\hline Número de parentes morando na vizinhança (nenhum) & 21,6 & 11,3 & 11,5 & 9,3 \\
\hline $\begin{array}{l}\text { Reconhece praticamente todos que passam na porta da } \\
\text { sua casa ( } \operatorname{sim} \text { ) }\end{array}$ & 8,0 & 12,6 & 13,1 & 21,3 \\
\hline Variáveis e escalas & \multicolumn{4}{|c|}{ Média (Desvio-padrão) } \\
\hline Tempo para caminhar até o final da vizinhança (minutos) & $6,1(0,22)$ & $13,5(0,35)$ & $19,8(0,78)$ & $29,2(1,26)$ \\
\hline Idade (anos) & $44,7(0,35)$ & $44,9(0,57)$ & $41,1(0,92)$ & $43,2(0,74)$ \\
\hline Tempo de moradia no domicílio (anos) & $14,8(0,26)$ & $16,8(0,44)$ & $16,2(0,71)$ & $16,6(0,60)$ \\
\hline Indicador econômico nacional & $586,8(4,06)$ & $601,6(6,6)$ & $582,8(11,4)$ & $601,4(9,0)$ \\
\hline Qualidade de serviços & $5,6(0,05)$ & $5,8(0,06)$ & $5,8(0,04)$ & $5,7(0,10)$ \\
\hline Aspecto estético & $5,4(0,11)$ & $5,6(0,14)$ & $5,6(0,22)$ & $5,7(0,17)$ \\
\hline Mobilidade & $6,8(0,10)$ & $7,2(0,09)$ & $7,2(0,11)$ & $7,5(0,07)$ \\
\hline Segurança & $4,2(0,07)$ & $4,1(0,12)$ & $3,9(0,20)$ & $4,1(0,15)$ \\
\hline Coesão social & $7,7(0,09)$ & $8,1(0,12)$ & $8,1(0,20)$ & $8,0(0,19)$ \\
\hline Participação na vizinhança & $8,8(0,14)$ & $9,3(0,21)$ & $9,9(0,23)$ & $9,0(0,26)$ \\
\hline Desordem física & $5,8(0,11)$ & $5,4(0,16)$ & $5,5(0,24)$ & $5,2(0,18)$ \\
\hline Desordem social & $4,9(0,10)$ & $5,1(0,16)$ & $5,2(0,22)$ & $5,2(0,21)$ \\
\hline Problemas na vizinhança & $7,4(0,13)$ & $7,4(0,19)$ & $7,6(0,28)$ & $7,4(0,25)$ \\
\hline Participação social & $4,7(0,08)$ & $4,7(0,08)$ & $5,1(0,19)$ & $4,4(0,14)$ \\
\hline
\end{tabular}

Adaptado de Friche et al. 22.

* 1: consideram as residências mais próximas da sua e o final deste quarteirão; 2: consideram os 5 quarteirões mais próximos; 3: consideram os 10 quarteirões mais próximos de sua residência e 4: consideram mais de 10 quarteirões da sua residência, seu bairro/comunidade/conjunto habitacional e bairros mais próximos.

aspectos estéticos (OR = 1,06; IC95\%: 1,01-1,13); avaliação positiva da mobilidade no interior da vizinhança (OR = 1,20; IC95\%: 1,12-1,30); identificação mais frequente de problemas na vizinhança (barulho, violência, consumo de drogas, entre outros) $(\mathrm{OR}=1,07$; IC95\%: 1,03-1,11); menor envolvimento em atividades sociais/lazer (OR = 0,93; IC95\%: 0,86-0,99); maior número de parentes morando na vizinhança $(\mathrm{OR}=4,03$; IC95\%: 2,55-6,38) e conhecer maior número de pessoas na vizinhança $(\mathrm{OR}=2,68$; IC95\%; 1,22-5,85). As demais variáveis testadas não apresentaram associação estatisticamente significante com a percepção da extensão territorial da vizinhança.

\section{Discussão}

Neste estudo, determinamos e quantificamos as características individuais associadas à percepção da extensão territorial da vizinhança. A maioria dos participantes $(57,8 \%)$ considerou a sua vizinhança como as residências mais próximas à sua e até o final do quarteirão. Esse resultado se assemelha ao relatado para Los Angeles, Estados Unidos. Os autores mediram a percepção da vizinhança utilizando escala ordinal, e $60 \%$ dos respondentes definiram sua vizinhança como aproximadamente dois quarteirões ou menos em qualquer direção ${ }^{18}$. Por outro lado, estudos que utilizaram mapas para essa avaliação reportam vizinhanças bem mais amplas, indicando 
Tabela 2

Distribuição de dispersão de escalas selecionadas de percepção do entorno físico e social da vizinhança para 4.048 participantes. Estudo Saúde em Beagá, Belo Horizonte, Minas Gerais, Brasil, 2008-2009.

\begin{tabular}{lcccccc}
\hline Escalas & $\begin{array}{c}\text { Número } \\
\text { de itens } \\
\text { na escala }\end{array}$ & $\mathbf{n}$ & $\begin{array}{c}\text { Componente 1 } \\
\text { (mínimo- } \\
\text { máximo) }\end{array}$ & $\begin{array}{c}\text { Componente 1 } \\
\text { Média (DP) }\end{array}$ & $\begin{array}{c}\text { Alfa de } \\
\text { Cronbach }\end{array}$ & $\begin{array}{c}\text { Número de } \\
\text { componentes } \\
\text { selecionados }\end{array}$ \\
\hline Qualidade de serviços & 8 & 3.647 & $2,0-9,8$ & $5,8(1,0)$ & 0,65 & 4 \\
Aspecto estético & 4 & 3.922 & $1,8-7,4$ & $5,5(2,0)$ & 0,60 & 2 \\
Mobilidade & 7 & 3.802 & $2,2-8,4$ & $7,1(1,4)$ & 0,51 & 3 \\
Segurança & 3 & 3.983 & $1,4-5,6$ & $4,2(1,41)$ & 0,53 & 3 \\
Coesão social & 6 & 3.415 & $2,4-9,7$ & $7,7(1,7)$ & 0,76 & 3 \\
Desordem física & 8 & 3.845 & $2,6-10,4$ & $5,4(2,1)$ & 0,62 & 3 \\
Desordem social & 6 & 3.655 & $1,9-7,5$ & $4,9(2,2)$ & 0,74 & 3 \\
Problemas na vizinhança & 16 & 3.571 & $2,7-12,3$ & $7,3(2,7)$ & 0,73 & 3 \\
Participação social & 7 & 4.030 & $2,3-9,0$ & $4,4(1,5)$ & 0,65 & 3 \\
\hline
\end{tabular}

DP: desvio-padrão.

Adaptado de Friche et al. 22 .

Nota: informações faltantes até, no máximo, 15,6\% para a escala coesão social.

Tabela 3

Variáveis que permaneceram no modelo final de regressão logística ordinal, modelo de chances proporcionais. Estudo Saúde em Beagá, Belo Horizonte, Minas Gerais, Brasil, 2008-2009 ( $n=3.094)$.

\begin{tabular}{lccc}
\hline Variável & OR & IC95\% & Valor de p \\
\hline Idade * & 0,99 & $0,99-1,00$ & 0,80 \\
Sexo (feminino) & 0,85 & $0,71-1,01$ & 0,07 \\
Indicador econômico nacional ** & 1,09 & $1,08-1,34$ & 0,00 \\
Tempo de moradia na mesma casa * & 1,00 & $0,99-1,01$ & 0,11 \\
Trabalha (sim) & 1,32 & $1,07-1,61$ & 0,00 \\
Número de parentes que residem na vizinhança (maioria) & 4,03 & $2,55-6,38$ & 0,01 \\
Número de pessoas que conhece na vizinhança (todos) & 2,68 & $1,22-5,85$ & $1,01-1,13$ \\
Aspecto estético & 1,06 & $1,12-1,30$ & 0,02 \\
Mobilidade & 1,20 & $1,03-1,11$ & 0,00 \\
Problemas na vizinhança & 1,07 & $0,86-0,99$
\end{tabular}

IC95\%: intervalo de 95\% de confiança; OR: odds ratio.

* Idade, tempo de moradia na mesma casa em anos. Categorias de referência: mulheres, trabalha, a maioria dos parentes, conhece todos;

** Para incremento de 200 pontos do indicador econômico nacional.

provável influência da estratégia para acessar a extensão da vizinhança percebida 12,15,16. É possível que a visualização da vizinhança em mapa favoreça a identificação de áreas que poderiam não ser lembradas pelo respondente utilizando questionário. O mapa pode levar o participante a delimitar sua vizinhança como mais extensa. Assim como observado em outro estudo 15, os resultados deste trabalho indicam que grupos de quarteirões aproximam-se mais da vizinhança 
autopercebida quando comparados a unidades administrativas como, por exemplo, o setor censitário. Devemos ressaltar que, na literatura consultada, as diferentes metodologias de mensuração da extensão territorial da vizinhança autopercebida dificultam possíveis comparações.

Não encontramos associação entre sexo e a percepção da extensão da vizinhança, o que é coerente com a maioria dos estudos 15,16,18. No entanto, nosso resultado deve ser analisado com cautela, uma vez que encontramos valor de p limítrofe $(0,07)$ para associação entre sexo feminino e percepções menos amplas da vizinhança.

A associação entre indivíduos mais jovens e percepções de vizinhança mais extensa, relatadas em alguns estudos 15,17, não foi verificada no presente trabalho. Era de se esperar que indivíduos mais velhos, menos ativos, relatassem vizinhanças menores; no entanto, essa associação não foi encontrada nem mesmo na análise univariada.

A associação entre posição socioeconômica menos vulnerável e percepções maiores de vizinhança encontradas neste estudo é coerente com outros dois trabalhos 15,18. As desigualdades resultantes de posições socioeconômicas heterogêneas podem resultar em diferentes percepções dos aspectos positivos e negativos da vizinhança. Indivíduos com maior escolaridade e renda podem ter maior discernimento em avaliar a qualidade dos serviços prestados na sua vizinhança ou mesmo capacidades distintas de reivindicar por melhores condições de moradia, o que pode favorecer a conservação de espaços coletivos e percepções diferenciadas da extensão da vizinhança.

O tempo de moradia na mesma residência é variável frequentemente incluída em estudos sobre a percepção da extensão territorial da vizinhança. Não encontramos associação entre tempo de residência e percepção mais extensa de vizinhança. Os resultados relatados na literatura são conflitantes 15,17,18,16. No entanto, é plausível que moradores que residem a mais tempo na vizinhança conheçam mais pessoas, tenham mais amigos e estejam mais engajados em sua comunidade, resultando em percepção mais ampla de sua vizinhança.

A convivência com maior número de parentes e amigos e o reconhecimento de maior número de pessoas no interior da vizinhança foram relacionados com percepções mais extensas, o que também foi relatado em dois outros estudos 17,18 . Esse resultado pode estar relacionado com o próprio conceito de vizinhança utilizado: “...Pode-se entender como vizinhança também o local onde o(a) sr.(a) reconhece a maioria das pessoas...”. Ainda, vizinhança não é apenas um conceito territorial, mas também social 26 , e a percepção da sua extensão está relacionada com o número maior de parentes, amigos e colegas na mesma vizinhança, assim como a presença de locais no interior da vizinhança que favoreçam a interação entre os moradores 27 .

Estar trabalhando esteve associado a percepções mais amplas de vizinhança, o que não foi encontrado na literatura consultada. É possível que aqueles atualmente empregados tenham maior rede de contatos. Essa rede de contatos pode, parcialmente, incorporar a vizinhança, uma vez que o trajeto da residência para o trabalho pode favorecer o contato com moradores próximos, ampliando sua percepção da vizinhança. Deve-se ressaltar que $80,5 \%$ dos participantes deste estudo trabalham em locais que não a sua vizinhança.

A interpretação dos resultados das escalas deve ser feita com base na análise do comportamento do seu escore. A escala aspecto estético recebe valores mais altos em seu escore quando a percepção do indivíduo é favorável em relação a áreas verdes, ausência de lixo ou entulho e locais agradáveis para crianças e jovens. Essa escala esteve associada com maior percepção da extensão territorial da vizinhança. Esses resultados podem ser comparados a outro estudo 16 , no qual indivíduos que relataram mais frequentemente características estéticas desfavoráveis perceberam a vizinhança como menos extensa. A escala problemas na vizinhança, que reporta valores mais altos para aquelas percebidas como vulneráveis (ruas e calçadas com conservação comprometida, barulho, pouca segurança, consumo de drogas, vandalismo e violência, entre outras), também esteve associada a percepções maiores de vizinhança. Esse resultado, aparentemente contraditório, demanda avaliação específica.

Na literatura consultada, nenhum estudo avaliou a relação entre mobilidade e percepção da extensão territorial da vizinhança. Neste estudo, indivíduos que reportaram sua vizinhança como um local que facilita o deslocamento a pé e caminhadas perceberam mais amplamente a sua vizinhança. É plausível que uma área que estimule a mobilidade das pessoas, atuando como facilitadora para a prática de atividades diversas no interior da vizinhança, também favoreça percepção mais ampla dela.

Os resultados da escala de participação social indicam que indivíduos mais engajados nessas atividades têm uma menor percepção da extensão da vizinhança. Esse resultado mostra-se inicialmente contraditório, uma vez que atividades como esporte/lazer podem facilitar maiores contatos na sua área de moradia. É possível que essas atividades não aconteçam necessariamente no interior da vizinhança. 
Algumas limitações deste estudo devem ser mencionadas. Como o objetivo foi a identificação dos fatores individuais associados à extensão territorial da vizinhança autopercebida, não foram considerados outros níveis hierárquicos na análise, uma vez que as características contextuais podem influenciar a percepção do tamanho da vizinhança 12,18. O trabalho de Santos et al. 28 utilizou dados contextuais para a construção dos limites da vizinhança, valendo-se, para isso, de método que considera a homogeneidade socioeconômica baseada em quatro indicadores (renda, educação, pessoas por domicílio e percentual de pessoas na faixa etária de 0-4 anos de idade) e pode ser consultado como alternativa às unidades administrativas e àquelas referenciadas na percepção do indivíduo. O fato de os participantes deste estudo serem maiores de 18 anos remete para possível objeto de estudo: crianças e adolescentes podem ter uma percepção de extensão de vizinhança diferente daquela dos adultos?

Algumas vantagens deste estudo devem ser mencionadas. Ele é pioneiro no Brasil quanto ao tema extensão territorial da vizinhança. Também utiliza estratégia não referenciada em unidades administrativas para definição de vizinhança. A população de estudo representa amostra probabilística daquela residente em um grande e complexo centro urbano, situado na terceira maior região metropolitana do país.

\section{Considerações finais}

Neste estudo, identificamos características individuais que podem contribuir para o entendimento do conceito de vizinhança. Diversas críticas têm sido feitas sobre o uso de unidades administrativas como aproximação de vizinhança 29,30,31,32, uma vez que indivíduos diferentes são expostos de maneira heterogênea aos fatores contextuais 14 . Os resultados deste estudo podem contribuir para estudos futuros objetivando estimar o(s) impacto(s) da vizinhança na saúde das populações ali aninhadas. Informações sobre as diferenças individuais na percepção da extensão territorial da vizinhança podem contribuir na construção de limites de vizinhança mais próximos da percepção dos indivíduos e unidades de análise mais sensíveis e representativas.

\section{Resumen}

El estudio trata sobre el creciente interés en investigación por los impactos en salud del vecindario. $\mathrm{Pa}$ ra definir vecindario hay dos estrategias principales: una utilizar fronteras administrativas, la otra tiene en cuenta la percepción subjetiva del individuo. El objetivo de este estudio fue identificar los factores asociados a la heterogeneidad de la percepción subjetiva de la dimensión territorial del vecindario entre los participantes en una encuesta de salud en Belo Horizonte, Minas Gerais, Brasil. Para verificar esta asociación se utilizó la regresión logística ordinal. Percepciones del vecindario más grandes se asocian con: mejor situación socioeco- nómica, el trabajo, la evaluación positiva de los aspec tos estéticos de la vecindad y la movilidad en el barrio, una mayor identificación de los problemas del barrio, una menor participación en actividades/pasatiempos deportivos y conocer a tantas personas en el barrio. Los resultados de este estudio pueden ayudar a definir los vecindarios más cercanos a la percepción de las personas, proporcionando estimaciones más precisas de los efectos del vecindario en la salud.

Salud Urbana; Comunidades Pequeñas;

Autopercepción 


\section{Colaboradores}

F. A. Célio contribuiu na concepção do projeto; interpretação dos dados e redação do artigo. C. C. Xavier, W. T. Caiaffa e A. A. L. Friche colaboraram na elaboração do instrumento; supervisão do trabalho de campo; operacionalização do instrumento; análise e interpretação dos dados; redação e revisão crítica do artigo e aprovação da versão final. A. C. Souza e V. P. Camargos participaram na análise e interpretação dos dados; redação e revisão crítica do artigo e aprovação da versão final. M. G. Cortês colaborou na interpretação dos dados; revisão crítica do artigo e aprovação da versão final. F. A. Proietti contribuiu na concepção do projeto e elaboração do instrumento; supervisão do trabalho de campo; operacionalização do instrumento; interpretação dos dados; revisão crítica do artigo e aprovação da versão final.

\section{Agradecimentos}

Aos professores, pesquisadores, alunos de graduação e pós-graduação, bem como aos funcionários administrativos do Observatório de Saúde Urbana de Belo Horizonte. À Secretaria Estadual de Saúde de Minas Gerais. Este estudo foi parcialmente financiado pelo CNPq, Fapemig e Ministério da Saúde.W.T. Caiaffa e F.A. Proietti são bolsistas de pesquisa do CNPq.

\section{Referências}

1. Schaefer-McDaniel N, Caughy MO, O'Campo P, Gearey W. Examining methodological details of neighbourhood observations and the relationship to health: a literature review. Soc Sci Med 2009; 70:277-92.

2. Diez Roux AV, Merkin SS, Arnett D, Chambless L, Massing M, Nieto FJ, et al. Neighborhood of residence and incidence of coronary heart disease. $\mathrm{N}$ Engl J Med 2001; 345:99-106.

3. Cohen D, Spear S, Scribner RD, Kissinger P, Mason K, Wildgen J. "Broken windows" and the risk of gonorrhea. Am J Public Health 2000; 90:230-6.

4. Mair C, Diez-Roux AV, Galea S. Are neighbourhood characteristics associated with depressive symptoms? A review of evidence. J Epidemiol Community Health 2008; 62:940-6.

5. Kim D. Blues from the neighborhood? Neighborhood characteristics and depression. Epidemiol Rev 2008; 30:101-17.
6. Subramanian SV, Chen JT, Rehkopf DH, Waterman PD, Krieger N. Racial disparities in context: a multilevel analysis of neighborhood variations in poverty and excess mortality among black populations in Massachusetts. Am J Public Health 2005; 95:260-5.

7. Chaix B, Kestens Y, Bean K, Leal C, Karusisi N, Meghiref K, et al. Cohort profile: residential and non residential environments, individual activity space and cardiovascular risk factor and diseasesThe RECORD Cohort Study. Int J Epidemiol 2012; 41:1283-92.

8. Ludwig J, Duncan GJ, Gennetian LA, Katz LF, Kessler RC, Kling JR, et al. Neighborhood effects on the long-term well-being of low-income adults. Science 2012; 337:1505-10.

9. Mujahid MS, Diez Roux AV, Shen M, Gowda D, Sánchez B, Shea S, et al. Relation between neighborhood environments and obesity in the multiethnic study of atherosclerosis. Am J Epidemiol 2008; 167:1349-57. 
10. Proietti FA, Oliveira CDL, Ferreira FR, Ferreira AD Caiaffa WT. Unidade de contexto e observação social sistemática em saúde: conceitos e métodos. Physis (Rio J.) 2008; 18:469-82.

11. Campbell E, Henly RJ, Irwin K. Subjective constructions of neighborhood boundaries: lessons from a qualitative study of four neighborhoods. J Urban Aff 2009; 31:461-9.

12. Coulton CJ, Korbin J, Chan T, Su M. Mapping residents' perceptions of neighborhood boundaries: a methodological note. Am J Community Psychol 2001; 29:371-83.

13. Chaix B, Kestens Y, Perchoux C, Karusisi N, Merlo J, Labadi K. An interactive mapping tool to assess individual mobility patterns in neighborhood studies. Am J Prev Med 2012; 43:440-50.

14. Chaix BC, Merlo J, Evans D, Leal C, Havard S. Neighborhood in eco-epidemiologic research: delimiting personal exposure areas. A response to riva, Gauvin, Apparicio and Brodeur. Soc Sci Med 2009; 69:1306-10.

15. Coulton CJ, Jennings MZ, Chan T. How big is my neighborhood? Individual and contextual effects on perceptions of neighborhood scale. Am J Community Psychol 2013; 51:140-50.

16. Haney W, Knowles E. Perception of neighborhoods by city and suburban residents. Hum Ecol 1978; 6:201-14

17. Guest AM, Lee BA. How urbanites define their neighborhoods. Popul Environ 1984; 7:32-56.

18. Pebley AR, Sastry N. Our place: perceived neighborhood size and names in Los Angeles. Los Angeles: California Center for Population Research, University of California; 2009.

19. Santos M. Metamorfoses do espaço habitado. Fundamentos teórico e metodológico da geografia. São Paulo: Editora Hucitec; 1988.

20. Camargos VP, César CC, Caiaffa WT, Xavier CC, Proietti FA. Imputação múltipla e análise de casos completos em modelos de regressão logística: uma avaliação prática do impacto das perdas em covariáveis. Cad Saúde Pública 2011; 27:2299-313.

21. Ferreira AD, César CC, Malta DB, Andrade ACS, Ramos CGC, Proietti FA, et al. Validade de estimativas obtidas por inquérito telefônico: comparação entre VIGITEL 2008 e inquérito Saúde em Beagá. Rev Bras Epidemiol 2011; 14:16-30.
22. Friche AL, Diez-Roux A, César C, Xavier C, Proietti F, Caiaffa W. Assessing the Psychometric and Ecometric Properties of Neighborhood Scales in Developing Countries: Saúde em Beagá Study, Belo Horizonte, Brazil, 2008-2009. J Urban Health 2013; 90:246-61.

23. Gerência de Epidemiologia e Informação, Secretaria Municipal de Saúde de Belo Horizonte. Índice de vulnerabilidade à saúde, 2003. http:/ / www.pbh. gov.br/smsa/biblioteca/gabinete/risco2003 (acessado em 18/Mar/2012).

24. Barros AJD, Victora C. Indicador econômico para o Brasil baseado no censo demográfico de 2000. Rev Saúde Pública 2005; 39:523-9.

25. Abreu MNS, Siqueira AL, Caiaffa WT. Regressão logística ordinal em estudos epidemiológicos. Rev Saúde Pública 2009; 43:183-94.

26. Chaskin R. Perspectives on neighborhood and community: a review of the literature. Soc Serv Rev 1997; 71:521-47.

27. Cross JE. Conceptualizing community attachment. In: Proceedings of the Rural Sociological Society Annual Meeting. Montreal: Rural Sociological Society; 2003. http://lamar.colostate.edu/ jecross/ pdf/presentations/Conceptualizing_Communi ty_Attachment_Cross_2003.pdf (acessado em 10/ Mai/2012).

28. Santos SM, Chor D, Werneck GL. Demarcation of local neighborhoods to study relations between contextual factors and health. Int J Health Geogr 2010; 9:34.

29. Chappell NL, Funk LM, Allan D. Defining community boundaries in health promotion research. Am J Health Promot 2006; 21:119-26.

30. Flowerdew R, Manley DJ, Sabel CE. Neighbourhood effects on health: does it matter where you draw the boundaries? Soc Sci Med 2008; 66: 1241-55.

31. Clapp JM, Wang Y. Defining neighborhood boundaries: are census tracts obsolete? J Urban Econ 2006; 59:259-84.

32. Diez-Roux AV, Mair C. Neighborhoods and health. Ann NY Acad Sci 2010; 1186:125-45.

Recebido em 10/Dez/2012

Versão final reapresentada em 09/Jan/2014

Aprovado em 19/Fev/2014 